\title{
NOTAS METODOLÓGICAS PARA EL ANÁLISIS DE LA MORTALIDAD DIFERENCIAL APLICACIÓN AL PARTIDO BONAERENSE DE TANDIL
}

\author{
POR \\ GUILLERMO A. VELÁZQUEZ
}

Planteamiento del problema

El reconocimiento de la relevancia de los elementos económicosociales como principal factor determinante en los procesos de cambio y diferenciación de la mortalidad ha tenido durante décadas poca atención por parte de los demógrafos, prevaleciendo una tradición de estudios «higienistas» y epidemiológicos propios del siglo XIX hasta hace relativamente poco tiempo.

De ahí que no exista aún un marco teórico-conceptual explícito y compartido por la comunidad científica de abordar esta problemática.

En un nivel descriptivo, la teoría de la transición demográfica parte de la experiencia de los países industrializados europeos en los últimos ciento cincuenta años y de otros no europeos en las últimas décadas. La sucesión de fases enunciadas no es más que un elemento constitituvo de la teoría más general de la transición demográfica. Su carácter eminentemente descriptivo ha hecho dudar de su validez como enfoque teórico, es decir, explicativo ${ }^{1}$.

${ }^{1}$ Chesnais, J. (1986): La transition démographique, París, INED, PUF.

Guillermo A. Velázquez: Departamento de Geografía. Universidad Nacional del CentroTandil (República Argentina).

Estudios Geográficos

Tomo LXI, 2000, n. ${ }^{\circ} 240$, julio-septiembre 
Paralelamente a la transición demográfica (descenso del nivel de mortalidad) tiene lugar la llamada «Transición epidemiológica» que supone un cambio cualitativo en la estructura según causas de muerte. Por este proceso se pasa entonces del predominio de causas «evitables» al de causas «no evitables», dependiendo la evitabilidad del estado actual de los conocimientos médicos de la sociedad y del nivel de democratización en su acceso.

Si tratamos de incrementar el nivel descriptivo a uno explicativo, esquematizando un poco las posturas, el debate sobre las causas del descenso de la mortalidad opone dos tendencias globales; por un lado los partidarios del predominio de las acciones de salud pública y por el otro los que privilegian la influencia del desarrollo económico y social.

La primera visión, dominante hasta la década de 1970 fue gestada en el marco del éxito obtenido con las políticas públicas de salud en Europa y, de modo más espectacular aún, en los países más pobres (campañas masivas de vacunación, erradicación de enfermedades endémicas, etc.). Este enfoque entonces - en su interpretación más extrema- aborda el problema con mucho optimismo, ya que bastaría con incrementar las camas de hospital, dotación de médicos, campañas sanitarias, etc., para alcanzar la reducción y la convergencia de los niveles de mortalidad entre los diferentes grupos sociales.

Como reacción a esta interpretación, a partir de mediados de los setenta cada vez más autores sostuvieron la idea de que los programas de asistencia sanitaria son claramente insuficientes y que solo el incremento del nivel de vida habría de jugar un rol determinante en la reducción y, fundamentalmente en la convergencia de la mortalidad entre los diferentes grupos sociales.

En el estado actual de nuestros conocimientos ninguna de las dos tesis puede ser aceptada como químicamente pura, ya que la oposición entre técnicas sanitarias y desarrollo socioeconómico supone una «falsa dicotomía» ${ }^{2}$, en la medida en que ninguno de los dos factores es independiente del otro. La mortalidad debe considerarse entonces como un fenómeno complejo y analizado, en consecuencia, desde una óptica multifactorial.

En síntesis, más allá de las controversias, resulta evidente que la muerte no constituye un fenómeno exclusivamente «natural» y que las

${ }^{2}$ Chesnais, op. cit. 
condiciones económicas y sociales (incluyendo también la acción del sistema sanitario) constituyen un factor preeminente en la determinación de los niveles de mortalidad de cada región y grupo social.

\section{Dificultades para el estudio de la mortalidad diferencial}

Si visualizamos a la mortalidad como un reflejo del contexto socioeconómico predominante, debemos pensar en factores tales como ocupación, nivel de instrucción, nutrición, vivienda, acceso a la asistencia médica, esparcimiento y otros elementos que conforman la calidad de vida. Como muchos de estos elementos se pueden comprar dependen, en gran medida, del nivel de ingresos. También deben considerarse las costumbres sociales, religión, artes y, en general, las expresiones culturales que influyen notablemente en las actitudes de los miembros de una sociedad, incluyendo aquellas que se relacionan con los hábitos higiénicos, preparación de alimentos, cooperación con los programas de salud pública, etc.

En primer lugar, muchos de estos elementos no son susceptibles de medición cuantitativa y, aunque pudiesen serlo no sería posible dimensionarlos en forma aislada por su interacción con los demás. Así, si relacionáramos mortalidad con ocupación, no sería posible aislar la influencia que están también ejerciendo el nivel de instrucción, tipo de vivienda, alimentación, etc.

En segundo término, no sería posible tratar experimentalmente con sociedades creando, por ejemplo un conjunto estable de circunstancias económicas para separar un segmento particular de un grupo social para someterlo a ellas. Además de las dificultades prácticas, habría obviamente objeciones éticas para realizar experimentos de esa índole.

En tercer término, la muerte es el resultado de efectos acumulados (diferentes ocupaciones, hábitats y otras circunstancias), cuyo resultado parece muy difícilmente separable y evaluable a lo largo del ciclo vital.

Señalemos por último que la información disponible adolece de mayores deficiencias que la de otros hechos vitales, máxime en el caso de los datos de base para estudios de mortalidad socialmente diferencial.

Teniendo en cuenta estas dificultades, la mejor forma de aproximación es intentar descubrir en la población segmentos realmente 
observables y que sean homogéneos respecto de factores específicos.

Existen, en principio, dos formas de definir segmentos de población. La primera (estructural) se basa en características económico-sociales de los individuos. Esto asegura una gran homogeneidad, pero no constituye una clasificación ecológica, ya que las personas con determinadas características pueden encontrarse geográficamente dispersas. La segunda manera (geográfica) considera segmentos espaciales (provincias, municipios) para los cuales se posee mayor riqueza de datos. Pero, frente a la ventaja de mayor información, la constitución de segmentos espaciales administrativos da resultados pobres desde el punto de vista de la homogeneidad interna; sobre todo porque la unidad espacial para la que existe información no ha sido diseñada para el análisis de factores socioeconómicos.

A medida que aumenta el nivel de análisis espacial, también lo hace la homogeneidad y significación social, pero disminuye drásticamente la información disponible. Así, los datos que pueden obtenerse fácilmente en el nivel nacional son más difíciles de hallar por provincias y mucho más aún con desagregación municipal o barrial. Lo mejor sería poder analizar unidades espaciales pequeñas y socialmente significativas. Con esto se obtendría una clasificación ecológica que cumpla a su vez con la necesidad de poseer una alta homogeneidad interna.

\section{El examen estructural de la mortalidad diferencial}

Categorías de análisis. La literatura demográfica sobre mortalidad diferencial cuenta con un abrumador predominio de estudios de países desarrollados aplicando categorías de análisis sociológicas o estructurales.

Si bien la variable explicativa central de estos estudios suele ser la categoría ocupacional, también se tienen en cuenta otros elementos tales como: nivel de instrucción, lugar de origen, sexo, estado civil y filiación, entre otros.

Es indudable que la categoría ocupacional puede proporcionar una excelente aproximación a la pertenencia social. Así en los estudios sobre mortalidad diferencial se comprueba, en líneas generales, que 
aquellos que pertenecen a categorías ocupacionales «bajas» (situados, por ende, en la base de la pirámide social) corresponde un mayor nivel de mortalidad que en el caso de los restantes grupos sociales. Sin embargo existen notorias dificultades para efectuar estudios de este tipo.

No resulta sencillo adscribir determinada ocupación con su correspondiente grupo social, ya que la información sobre ocupaciones adolece de serios problemas. Son frecuentes las descripciones genéricas, tales como «empleado», «comerciante», «jubilado», etc. Existe además una tendencia a manifestar ocupaciones de mayor prestigio relativo que la o las realmente ejercidas; esto es especialmente cierto en el caso de la declaración de ocupación efectuada por los deudos del difunto.

Otras categorías de aproximación tienen menores problemas de asignación que la ocupación, pero no son tan determinantes en la definición de la pertenencia social.

Así, si consideramos el nivel de instrucción, también encontraremos diferentes niveles de mortalidad. Los sectores con mayor nivel educativo poseen, en general, una menor mortalidad y viceversa. Si bien podemos pensar en el efecto positivo que ejerce la instrucción en sí misma, debemos tener en cuenta la asociación positiva que existe entre ella y la jerarquía ocupacional.

Analizado la situación migratoria vemos que el lugar de origen arroja ciertas diferencias en los niveles de mortalidad. Aquí, nuevamente, el contexto de formación inicial puede ejercer cierto efecto, pero también lo está haciendo la inserción ocupacional diferencial de los migrantes.

En el examen de la mortalidad diferencial según género también se obtienen indicios sobre diferencias sociales. Si bien la tendencia global refleja una mayor mortalidad masculina, en diferentes contextos sociales esta diferencia se hace más o menos significativa, llegando incluso a valores negativos (es decir, sobremortalidad femenina) en algunos casos.

El estado civil y la filiación son otras de las variables estructurales que suelen considerarse para el análisis de la mortalidad diferencial. En el caso de los casados - o unidos con pareja estable que quedan a veces subregistrados - se observa una menor mortalidad respecto de los solteros. A su vez la mortalidad infantil es mayor en los niños de origen extramarital. 


\section{El estudio geográfico de la mortalidad diferencial}

Los aspectos locacionales tienen un doble rol: por un lado pueden contribuir a expresar la situación económico-social (correlación residencia-estrato social), por el otro resultan - al menos en parte- uno de sus condicionantes (accesibilidad, condiciones de sitio, etc.).

Las similitudes en las dificultades para efectuar un análisis clásico «estructural» u otro de tipo «locacional» son manifiestas: en ambos casos ocupa un lugar muy importante el criterio de clasificación y operacionalización de las variables.

Para estudiar los diferenciales de mortalidad de la población a partir de elementos de tipo estructural, resulta necesario no incluir en la misma categoría ocupacional a profesiones de desigual jerarquía. En este punto podría hacerse una analogía afirmando que para efecturar un adecuado análisis geográfico o «locacional» es preciso que haya un cierto grado de conformidad en las unidades espaciales que se van a utilizar.

Construcción de las unidades de análisis. En nuestro estudio sobre las diferencias de mortalidad de la población del partido de Tandil ${ }^{3}$ hemos comprobado diferencias de mortalidad significativas. Tandil superó los 90.000 habitantes en el censo de 1991, correspondiendo más de 80.000 a la planta urbana.

El partido está situado en el centro-sur de la provincia de Buenos Aires, posee una estructura económica y social diversificada, ya que es cabecera de una rica zona agrícola y ganadera que también posee canteras de granito. Su desarrollo industrial, que data de principios del siglo xx está sesgado por la industria metalúrgica. El componente más dinámico de su economía es el sector terciario, fundamentalmente el vinculado con la actividad turística y el papel dinamizador que ejerce la presencia de una de las pocas universidades del interior de la provincia. Por la diversificación de su estructura económica y social ha sido considerada en numerosas oportunidades como «ciudad testigo».

Para analizar las diferencias de mortalidad de la población de Tandil, hemos sectorizado el área urbana considerado la distribución espacial de los siguientes aspectos:

${ }^{3}$ Velázquez, G. (1993): Desigualdades geo-sociales de la mortalidad. El caso del Partido de Tandil (Buenos Aires). Tesis doctoral UBA, Buenos Aires. 
- Porcentaje de población con necesidades básicas insatisfechas.

- Infraestructura de servicios urbanos.

- Consideración social de los «Barrios».

- Distribución de los programas de ayuda alimentaria y

- Categoría predominante de vivienda.

Las unidades resultantes. Los elementos anteriormente señalados se distribuyen heterogéneamente en la planta urbana de Tandil. En general puede observarse una clara dicotomía entre el centro y la periferia urbana, siendo posible distinguir, a su vez, desigualdades entre los diferentes «tentáculos» de expansión.

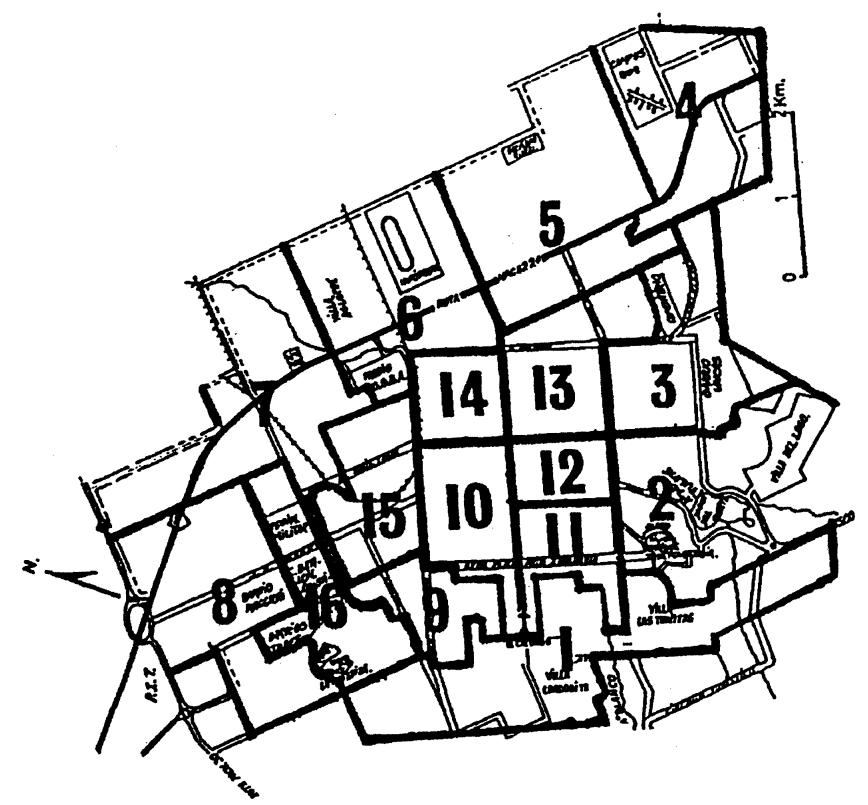

Fig. 1. Sectorización de Tandil.

Referencias: 1) Las Tunitas, Villa Cordobita. 2) Villa del Parque, Brisas de Lago, Villa del Lago. 3) Villa Manantial, Falucho I, Ceferino Namuncurá. 4) Barrio Golf, área rururbana. 5) Villa Gaucho, Barrio Güemes, área rururbana. 6) Barrios Palermo, La Tandilera, Villa Alduncin, 25 de Mayo, San Francisco, Vila Aguirre, Fatica. 7) Matadero, Villa Iatalia (N) área rururbana. 8) El Tropezón, La Movediza, área rururbana. 9) Barrio Metalúrgico, Atepam I y II, General Belgrano, Villa Laza. 10) Zona Hospital. 11) Zona Cuatro Avenidas (0) (semicéntrica). 12) Zona Cuatro Avenidas (E) (céntrica. 13) Zona Terminal Omnibus. 14) Zona Estación FCC. 15) Villa Italia (s), Villa Galicia, La Florida. 16) Barrio de Oficiales y Suboficiales. 
A partir de la consideración de estos aspectos proponemos una sectorización que incluye 16 zonas urbanas y que responde al propósito de obtener unidades espaciales adecuadas para realizar un análisis de tipo locacional de los diferenciales de mortalidad de la población.

\section{Resultados}

El cuadro I muestra la $e_{(0)}$, TMI y la estructura ocupacional de cada una de las 16 zonas precedentemente definidas.

\section{CUADRo I}

EXPECTATIVA DE VIDA, TASA DE MORTALIDAD INFANTIL, ESTRUCTURA OCUPACIONAL Y POBLACIÓN TOTAL POR ZONAS. TANDIL (1980-1985)

\begin{tabular}{rrrrr}
\hline Zona & $\mathbf{e}_{(\mathbf{0})}$ & TMI & $\begin{array}{c}\text { \% pobl. } \\
\text { ocup. } \ll \text { » }\end{array}$ & $\begin{array}{c}\text { Población } \\
\text { total }\end{array}$ \\
\hline 1 & 66,57 & 37,8 & 81,8 & 2.849 \\
2 & 70,06 & 22,2 & 38,1 & 2.562 \\
3 & 71,74 & 14,9 & 26,7 & 3.404 \\
4 & 72,64 & 11,0 & 66,7 & 2.119 \\
5 & 66,88 & 36,4 & 33,3 & 2.666 \\
6 & 70,59 & 19,9 & 57,8 & 5.243 \\
7 & 68,95 & 27,1 & 70,4 & 6.028 \\
8 & 70,06 & 22,2 & 63,6 & 1.278 \\
9 & 70,38 & 20,8 & 28,6 & 4.714 \\
10 & 70,91 & 18,5 & 32,0 & 10.747 \\
11 & 71,37 & 16,5 & 40,0 & 5.701 \\
12 & 72,04 & 13,6 & 20,2 & 6.987 \\
13 & 73,34 & 8,0 & 37,0 & 10.096 \\
14 & 73,20 & 8,6 & 47,2 & 7.012 \\
15 & 72,29 & 12,5 & 31,9 & 9.531 \\
16 & 73,43 & 7,6 & 0,0 & 1.616 \\
Total & & & & \\
& 70,02 & 25,2 & 43,8 & 82.553
\end{tabular}

Referencias $\mathrm{e}_{(\mathrm{o})}$ Expectativa de vida al nacer, TMI Tasa de mortalidad infantil, \% pobl. ocup. «B» porcentaje de población fallecida con ocupación «baja» (estratos ocupacionales 7, 8 y 9 de la clasificación de la OIT adaptada a la Argentina por Sautu, 1979).

Fuente: Registro Civil y elaboración personal. 
Podemos notar que las unidades de localización utilizadas establecen importantes diferencias en los indicadores de mortalidad. La correspondencia de mayores niveles en aquellas zonas con peor composición ocupacional nos muestra que el factor espacial no actúa en forma autónoma: existe una correspondencia entre localización y ocupación.

Llegados a este punto nos parece pertinente proponer una instancia que tienda a superar las limitaciones de estos dos enfoques aisladamente considerados.

\section{Hacia un estudio integral de la mortalidad diferencial}

Si bien la magnitud de los diferenciales de mortalidad obtenidos mediante un análisis puramente estructural o exclusivamente locacional resulta importante, aquellos son producto de una interacción, y como tal deben ser analizados. En otros términos este análisis puede avanzar al menos un paso más si consideramos en forma conjunta los aspectos geográficos con los inherentes a la estratificación social, aproximados a partir de la jerarquía ocupacional.

Aunque, cuantitativamente hablando, la mayoría de la población que pertenezca a jerarquías ocupacionales elevadas tenderá a vivir en aquellos lugares mejor categorizados (y viceversa), no podemos dejar de señalar la existencia de una amplia gama de situaciones posibles.

Por un lado pueden existir desfases entre categoría de residencia $y$ jerarquía ocupacional. Así es posible encontrar, por ejemplo, personal doméstico y de servicios que «vive» en residencias de categoría o profesionales (veterinarios, médicos, etc.) que residen, quizás transitoriamente, en áreas relativamente marginales.

Por el otro, este doble análisis puede servir para mejorar el control de la categoría ocupación respecto del concepto del grupo social. Así, en la clasificación utilizada, los «profesionales» (en abstracto) están genéricamente categorizados en la jerarquía ocupacional alta pero, como resulta obvio, esto no refleja la realidad en todos los casos, ya que un profesional puede ser empleador, productor familiar, empleado, trabajador por cuenta propia, desocupado, etc. Nuestra hipótesis es que parte de esta heterogeneidad social puede mejorarse al controlar las ocupaciones en función de la re- 
sidencia. Es decir que si existen grupos de profesionales con niveles muy diferentes de patrimonio o remuneración, su lugar de residencia tenderá a diferir ya que, en realidad, pertenecen a diferentes estratos sociales.

En otros términos, el cruce de aspectos sociológicos y geográficos permite una mejor definición de los grupos sociales y un dimensionamiento más claro de sus diferenciales. Debemos tener presente, no obstante, que la variable explicativa central de las diferencias de mortalidad de la población debe buscarse en la estructural social en su conjunto, por lo que lo expuesto en los dos párrafos anteriores no implica «fetichizar» al espacio.

El resultado de cruzar tres jerarquías ocupacionales y tres categorías de localización (reagrupando en tres categorías las 16 zonas originales por razones de consistencia estadística) es, lógicamente, una matriz de $3 \times 3$, cuya diagonal principal tiene la mayor cantidad de casos:

\section{CuAdro II}

POBLACIÓN FALLECIDA SEGÚN JERARQUÍA OCUPACIONAL Y CATEGORÍA DE LOCALIZACIÓN. TANDIL, 1980-1985

\begin{tabular}{lcccc}
\hline \multicolumn{1}{c}{$\begin{array}{c}\text { Jerarquía } \\
\text { ocupacional }\end{array}$} & Alta & Media & Baja & $\begin{array}{c}\text { Total } \\
\text { ocupaciones }\end{array}$ \\
$\begin{array}{l}\text { Categoría } \\
\text { localización }\end{array}$ & 174 & 690 & 259 & 1.123 \\
\hline $\begin{array}{l}\text { Media-alta } \\
\text { (zonas } 3,10,12,15 \text { y 16) }\end{array}$ & 82 & 665 & 370 & 1.117 \\
$\begin{array}{l}\text { Media } \\
\text { (zonas 2, 5, 9, 11, 13 y 14) }\end{array}$ & 9 & 120 & 178 & 307 \\
$\begin{array}{l}\text { Baja } \\
\text { (zonas 1, 4, 6, 7 y 8) }\end{array}$ & 301 & 1.543 & 960 & 2.804 \\
\hline Total 16 zonas & & & &
\end{tabular}

Nota: de los 5.530 fallecidos entre 1980 y 1985, sólo 3.200 cumplen la doble condición de tener ocupación registrada y edad mayor a 30; 4.760 tienen localización definida y edad mayor a 30 y solamente 2.804 cumplen la triple condición de edad mayor a 30 , domicilio definido y ocupación registrada. Las zonas se corresponden con las de la figura 1.

Fuente: Registros Civiles, Caja Nacional de Previsión Social y elaboración personal. 
Desde un punto de vista integral la celda 1,1 incluye la verdadera «clase alta», ya que en ella coinciden las mejores ocupaciones y localizaciones; en contraposición la celda 3,3 abarca a la verdadera «clase baja». En el resto de las celdas se producen desfases más o menos importantes entre lo geográfico y lo sociológico que pueden responder tanto a situaciones reales como a problemas de información o clasificación.

\section{CuAdro III}

VIDA MEDIA SEGÚN JERARQUÍA OCUPACIONAL Y CATEGORÍA DE LOCALIZACIÓN. TANDIL, 1980-1985

\begin{tabular}{lcccc}
\hline \multicolumn{1}{c}{$\begin{array}{c}\text { Jerarquía } \\
\text { ocupacional }\end{array}$} & Alta & Media & Baja & $\begin{array}{c}\text { Total } \\
\text { ocupaciones }\end{array}$ \\
$\begin{array}{l}\text { Categoría } \\
\text { localización }\end{array}$ & 77,6 & 69,6 & 68,0 & 70,47 \\
\hline $\begin{array}{l}\text { Media-alta } \\
\text { (zonas 3, 10, 12, 15 y 16) }\end{array}$ & 77,6 & 69,0 & 67,7 & 69,20 \\
$\begin{array}{l}\text { Media } \\
\text { (zonas 2, 5, 9, 11, 13 y 14) }\end{array}$ & 74,7 & 63,7 & 62,6 & 63,38 \\
$\begin{array}{l}\text { Baja } \\
\text { (zonas 1, 4, 6, 7 y 8 }\end{array}$ & 76,53 & 68,73 & 66,30 & 68,74
\end{tabular}

Nota: de los 5.530 fallecidos entre 1980 y 1985, sólo 3.200 cumplen la doble condición de tener ocupación registrada y edad mayor a 30; 4.760 tienen localización definida y edad mayor a 30 y solamente 2.804 cumplen la triple condición de edad mayor a 30, domicilio definido y ocupación registrada. Las zonas se corresponden con las de la figura 1.

Fuente: Registros Civiles, Caja Nacional de Previsión Social y elaboración personal.

El cuadro nos muestra la interacción que establecen ocupación y localización en los niveles de mortalidad de la población. Tanto de derecha a izquierda como de la parte superior a la inferior podemos observar una notable gradiente. Las diferencias entre sus extremos son superiores a los de la jerarquía ocupacional o de categoría de localización separadamente consideradas debido a que esta matriz capta y controla mejor las posibles contradicciones entre ocupación, residencia y grupo social. 
Resulta pertinente señalar que la magnitud hallada además de estar subestimada por una serie de factores (ver el punto «dificultades para el estudio de la mortalidad diferencial) disminuye por el subregistro diferencial de la combinación de ocupaciones y localizaciones (ver nota del cuadro II): hacia la derecha y hacia la parte inferior del cuadro III, donde se espera una mortalidad mayor, el subregistro es también mayor.

\section{Conclusiones}

Cuando se intenta efectuar estudios sobre mortalidad diferencial, el paradigma demográfico ortodoxo tiene limitaciones, ya que no resulta posible aplicarlo a situaciones reales. A menos que nos conformemos con análisis de un nivel de generalización tal como: «la mortalidad sube», «la natalidad baja», etc., debemos dejar entre paréntesis cierto purismo matemático e intentar ir un poco más allá de lo global.

Pensamos que un adecuado análisis espacial, convenientemente articulado con indicadores de tipo estructural, puede abrir nuevas posibilidades para los estudios de mortalidad diferencial.

Los resultados obtenidos muestran que aún en una ciudad de menos de 100.000 habitantes es posible hallar diferencias de mortalidad substancial definiendo adecuadas categorías ocupacionales y locacionales y su interacción.

Si en lugar de las categorías ocupacionales definidas nos hubiésemos valido de otras clasificaciones (por ejemplo por ramas de actividad) o, en vez de las unidades de análisis territorial empleadas, hubiéramos utilizado fracciones censales preestablecidas, las diferencias existentes se hubiesen enmascarado bajo una supuesta homogeneidad: el típico problema de la Unidad Espacial Modificable.

Para el análisis de la mortalidad diferencial existe un fuerte desfase entre el marco teórico y las posibilidades reales de la investigación, fundamentalmente en lo que respecta a la confiabilidad, comparabilidad y disponibilidad de las fuentes. Volviendo a nuestro debate teórico inicial acerca de la incidencia que tienen sobre la mortalidad la acción del sistema sanitario y la estructura social, una supuesta homogeneidad, impediría identificar a los grupos de «riesgo», que resulta menester identificar si se pretende dar cierta racionalidad a la asignación de recursos. 
Dados los diferenciales obtenidos, es mucho lo que aún podría hacerse con pocos recursos desde el sistema socio-sanitario, pero indudablemente también se impone la necesidad de una sociedad menos cruel si el propósito es que la teoría de la convergencia se acerque un poco más a la realidad.

\section{BIBLIOGRAFÍA}

ACCINELli, M.; Müller, M. (1978): «Un hecho inquietante: la evolución reciente de la mortalidad en la Argentina», en Notas de Población, 17, Santiago de Chile, CELADE.

Buenos Aires (1991): Estadísticas vitales años 1988-1990, La Plata, Ministerio de Salud, Subdirección de Información Sistematizada.

ChESNAIS, J. (1986): La transition démographique, París, INED, PUF.

GABRIEL, K.; RONEN, I. (1974): «Estimación de la mortalidad a partir de las tasas de mortalidad infantil», San José, Costa Rica, CELADE.

George, P. (1974): Sociología y Geografía, Barcelona, Península.

MÜLler, M. (1984): «Mortalidad infantil y desigualdades sociales en Misiones». Buenos Aires, CENEP.

NACIONES UNIDAS (1986): Técnicas indirectas de estimación demográfíca, Manual X, Nueva York.

OIT, (1968): Clasificación Internacional Uniforme de Ocupaciones, Ginebra.

Ortega, A. (1987): Tablas de mortalidad, San José, Costa Rica, CELADE.

Otero, H.; Velázquez, G. (1995): Tablas de mortalidad de la Provincia de Buenos Aires (1980-1982). Partidos y Zonas Sanitarias. Tandil, SECyTUNC.

REYNAUD, A. (1979): «Le concept de classe Socio-Spatiale. La notion de region dans son contexte social». París, UE Letters et Sciences Humaines, Reims \# 38.

SAUTU, R. (1979): «Oportunidades ocupacionales diferenciales por sexo en la República Argentina». Buenos Aires, Cuadernos del CENEP, 10 Centro de Estudios de la Población.

VALLIN, J. (1993): L'avenir de l'espérance de vie, París, INED, PUF.

VelÁZquez, G. (1993): Desigualdades geo-sociales de la mortalidad. El caso del Partido de Tandil (Buenos Aires). Tesis doctorial UBA, Buenos Aires.

- (1997): ¿Por qué algunos viven más que otros? Tandil, Universidad Nacional del Centro de la Provincia de Buenos Aires.

RESUMEN: Los dos procedimientos habituales para realizar estudios sobre mortalidad diferencial (definición de grupos sociales a partir de la ocupación o a través de unidades espaciales administrativas), tienen dificultades. En este trabajo se propone y aplica un procedimiento alternativo que permite superar algunos de estos problemas, controlando los problemas de información, la interacción y los posibles desfases entre jerarquía ocupacional y lugar de residencia. Para ello se utilizan adecuadas unidades de análisis espacial y una matriz estructural-locacional. 
PALABRAS CLAVE: Mortalidad diferencial. Análisis estructural-locacional. Enfoque integral.

ABSTRACT: Two usual methods form aking studies about differential mortality (social groups definition from occupation or social groups definition by using administrative spatial units), both have problems. This paper poposes and applies an alternative way to overcome same problems, by managing information defects, the interaction between the occupational range and residential place and, its possible incoherences. In order to this, they are usec both adequate spatial study units and a structural-locational matrix.

KEY wORDS: Differential mortality. Structural-locational analysis. Integral view.

REsumé: Les deux procédés habituels pour réaliser des études sur la mortalité différentielle (définition de groupes sociaux à partir de categories professionnelles ou d'unités spatiales administratives) ont des certains de ces problémes, tout en contrôlant les problémes d'information et l'interaction et les possibles décalages entre hiérarchie proféssionnelle et lieu de résidence. Pour ce faire l'on utilise des unités d'analyse spatiale adéquates et une matrice structurelle-résidentielle.

MoTS CLÉ: Mortalité différentielle. Analyse structurelle-résidentielle. Approche intégrale. 